\title{
La récente sécheresse des régions sahéliennes
}

\author{
PAR
}

\section{Jacques Sircoulon}

\author{
Ingénieur Hydrologue, Office de li Resherche scientifique et Technique Outre-Mer
}

\section{Ampleur de la sécheresse et délimi- tation géographique}

Parmi les cataclysmes climatiques qui ont affecté notro planète au cours de ces dernières décennies, la sécheresse qui a frappé de 1968 à 1973 les deux bandes tropicales qui ceinturent le globe, tient une place prépondérante.

Remarquablement sévère dans les régions sahéliennes de l'Afrique de l'Ouest, cette sécheresse présente trois caractères dont la conjonction lui donne un aspect inhabituel : sa rigueur tout d'abord, son extension et surtout sa persistance pendant plusieurs années consécutives. Les effets de ce phénomène sur la vie des populations et sur léconomie des pays atteints sont malheureusement bien connus grâce aux moyens actuels d'information. L'opinion mondiale a efé depuis plusieurs années sensibilisée à l'ampleur de cette catastrophe et de nombreux organismes internationaux sont intervenus soit pour assister les populations, soit pour essayer de comprendre les causes de la sécheresse, étudier les risques de son retour et déterminer les ressources hydriques des pays concernés.

La zone de l'Afrique francophone au sud du Sahara, directement atteinte par la raréfaction des pluies, comprenc d'ouest en est les pays suivants : la Mauritanie et le Sénégal en totalité, la majeure partie du Mali et de la Haute-Volta, la totalité du Niger et la majeure partie du Tchad, soit un domaine de près de 5 millions de $\mathrm{km}^{2}$ pour une population de 25 millions d'habitants.

Directement en bordure du Sahara, dans la zone subdésertique qui reçoit moins de $300 \mathrm{~mm}$ de pluie en année moyenne, les effets de la sécheresse ont été les plus désastreux. Dans cette frange aride où l'équilibre entre l'homme et son environnement est toujours très précaire et où dono les conditions de vie sont toujours très dures, l'assèchement des points d'eau, la diminution des pâturages, l'hécatombe des troupeaux ont été spectaculaires et la situation des pasteurs nomades qui ont dû refluer plus au sud vers des régions également éprouvées est dramatique aujourd'hui encore, au point que certains se demandent si une certaine forme de nomadisme n'est pas en train de disparaitre.

Dans la zone sahélienne proprement dite, qui s'étend entre les isohyètes 300 et $750 \mathrm{~mm}$, et où les pluies sont concentrées sur 3 à 4 mois de l'année, les effets ont été presqu'aussi sérieux. Dans cette région où les paysans sédentaires coexistent avec les gardiens de troupeaux, les cultures traditionnelles d'irrigation ou de décrue n'ont pu être assurées, les barrages hydroagricoles se sont asséchés et neême les populations riveraines des grands fleuves Sénégal et Niger n'ont pu profiter des effets bénéfiques des inondations annuelles, les crues étant restées trop faibles. Plus à l'est, le lac Tchad, principalement alimenté par le Chari dont les apports ont été déficitaires de 1965 a 1974, a connu une baisse spectaculaire de son plan d'eau, entraînant sa coupure en deux cuvettes, puis l'assèchement complet de la cuvette nord en novembre 1975 .

La zone du climat tropical pur, comprise entre les isohyètes annuelles 750 et $1200 \mathrm{~mm}$ a été également largement touchée mais le déficit en eau y a eu des conséquences beaucoup moins dramatiques qu'au Sahel, malgré la rareté du phénomène observé car les ressources sont restées suffisantes en général pour assurer un minimum alimentaire aux populations.

Nous limitons notre étude de la sécheresse au seul Sahel, c'est-à-dire à la région qui, englobant les régimes subdésertiques et sahéliens, est bordée au sud par l'isohyète $750 \mathrm{~mm}$. Cette limite part du $14^{\mathrm{e}}$ degré de latitude 
nord sur la zone côtière du Sénégal, passe par Kaolack, puis s'infléchit progressivement vers le sud en passant par Segou au Mali, le nord de Ouagadougou en Haute-Volta et un peu au sud de N'Djamena au Tchad. Aux confins du Tchad et du Soudan, cette limite est légèrement inférieure à 12 degrés de latitude nord (fig. 1).
- en juillet-août, l'anticyclone saharien a fait place à la dépression saharienne et l'anticyclone de Sainte-Hélène est remonté vers le nord, la mousson envahit les régions sahéliennes.

Le contact entre les deux masses d'air est le front intertropical ou F.I.T. En janvier, le F.I.T. se trouve dans la

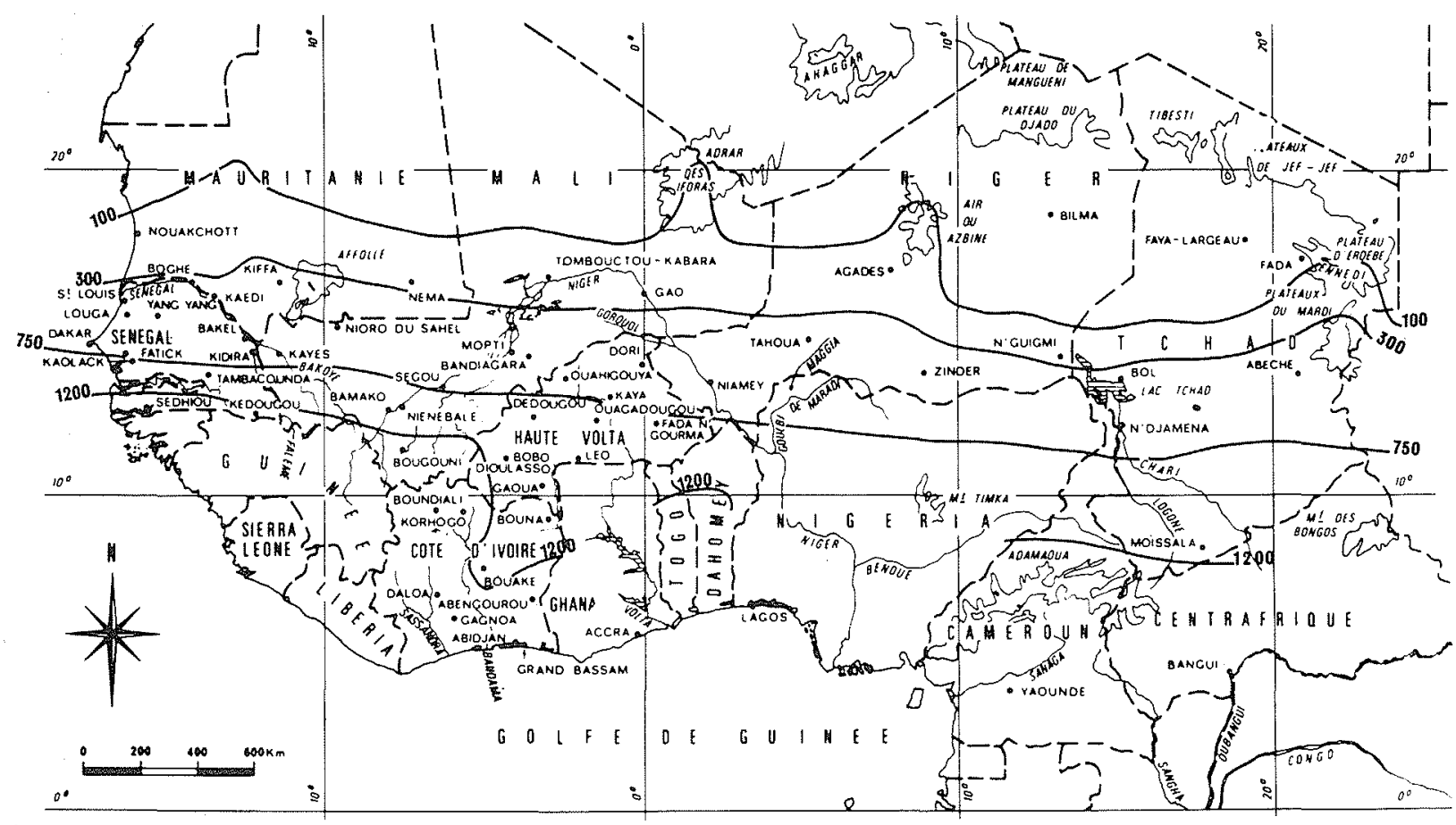

1/ Hauteurs de précipitations annuelles au Sahel (Valeurs médianes en $\mathrm{mm}$ )

\section{Caractéristiques hydroclimatiques du Sahel}

Afin de comprendre les particularités hydropluviométriques de cette sécheresse, un rappel des particularités du climat et de l'écoulement au Sahel sont nécessaires.

\subsection{Le régime des précipitations}

Les précipitations résultent pcur l'essentiel $\left({ }^{1}\right)$ de l'interaction de deux masses d'air :

- l'air tropical continental chaud et sec venant de l'est ou du nord-est et souvent désigné par le terme "harmattan",

- l'air équatorial maritime, originaire de l'anticyclone de Sainte-Hélène ; il vient du sud-ouest et est communément désigné par le nom de "mousson".

Ces deux masses d'air se déplacent au cours de l'année en accompagnant avec un certain décalage le soleil dans son oscillation apparente autour de l'équateur :

en janvier, l'anticyclone saharien occupe une position méridionale, l'harmattan souffle en permanence du nord-est ou du nord;

...............

(1) l'alizé boréal maritime provenant de l'anticyclone des Açores n'ayant une influence marquée qu'à l'ouest de la Mauritanie et du Sénégal. partie sud de la Côte-d'Ivoire, en août il est remonté jusque vers le vingtième parallèle. Au début de septembre, il redescend vers le sud pour rejoindre la position qu'il occupait en janvier. Au contact de ces masses d'air et dans la masse nuageuse de la mousson se produisent des formations orageuses donnant lieu à des précipitations.

A très grande échelle, ce phénomène simple d'oscillation de masses d'air permet de schématiser de façon commode la répartition spatio-temporelle des précipitations.

- Pour une latitude donnée, la durée des précipitations et leur hauteur annuelle sont fonction de la durée séparant la montée puis la descente du F.I.T. (donc, plus le F.I.T. remontera vers le nord et plus les précipitations seront excédentaires).

- Les isohyètes suivent la même direction que celle de la trace au sol du F.I.T. (avec un certain nombre d'irrégularités dues au relief ou à l'influence de zones marécageuses étendues).

Ainsi, en régime subdésertique, la saison des pluies ne dure que deux mois alors qu'en régime sahélien, elle s'étend sur trois à quatre mois, (le mois d'août étart dans ce régime le mois le plus arrosé), le restant de l'année ne comportant généralement aucune averse.

$\mathrm{Si}$ on se place à une échelle plus fine, on est malheureusement amené à constater qu'il n'existe pas qu'une seule variabilité du total annuel de pluie. Cette masse 
nuageuse n'est en effet pas homogène et il existe à l'intérieur de chaque saison des pluies, et d'une année à l'autre, une répartition irrégulière importante des précipitations dans le temps et dans l'espace. Cette irrégularité et la notion un peu trompeuse d'isohyète interannuelle augmentent d'autant plus que l'on est plus proche de la bordure du Sahara où un seul orage peut rendre une année excédentaire. Cette irrégularité temporelle des précipitations à l'échelle de l'année peut avoir des conséquences graves lorsqu'il s'agit de cultures non irriguées. Ainsi des pluies trop tardives ou au contraire trop précoces pourront avoir un effet désastreux sur les récoltes, de même que des années excédentaires présentant une mauvaise répartition mensuelle n'amèneront pas une augmentation des récoltes. L'irrégularité spatiale est tout aussi préjudiciable à l'efficacité des aménagements d'hydrauliques et rend difficile la détermination précise de la valeur moyenne pluviométrique en un point.

Une étude fine de l'évolution spatio-temporelle des pluies dans les zones climatiques concernées par la sécheresse se heurte là encore à d'autres difficultés:

- la densité des postes de mesure est en général assez moyenne et toujours très faible dans les régions les plus septentrionales, qui sont aussi celles où la sécheresse semble avoir été la plus forte;

- la qualité des relevés est très inégale, les erreurs de mesures, l'inadéquation de l'appareillage ou les variations dans l'environnement des stations étant fréquentes.

D'autre part, la comparaison entre la sécheresse actuelle et les autres sécheresses historiques est rendue très délicate par la rareté des observations disponibles avant les années 1920-1930. Si la sécheresse de la période 19401944 est assez bien connue, il n'en va pas du tout de même de celle de la période 1907-1914, beaucoup plus rigoureuse, pour laquelle on ne compte qu'une dizaine de postes pluviométriques en activité pour toute la zone sahélienne dont les observations soient utilisables.

\subsection{Régime des cours d'eau originaires du Sahel}

Dans les régions sahéliennes, la notion de superficie d'un bassin versant n'a pas grande signification, la dégradation hydrographique est en effet la règle et les phénomènes d'endoréisme sont fréquents. La notion traditionnelle de croissance du débit de l'amont vers l'aval ne peut donc s'appliquer, l'écoulement peut n'affecter qu'une faible partie d'un bassin versant, il dépendra étroitement du type de l'aire d'alimentation : zone dégradée ou non, forte pente ou non, sol imperméable ou non.

D'une façon générale, l'hydrogramme annuel des débits se présente comme une série de pointes de crues : les premières au début de juillet, les dernières en septembre. Après chaque crue, l'écoulement cesse sauf lorsque le sol est perméable. Dans ce cas, l'écoulement peut être permanent jusqu'en octobre.

En zone subdésertique, quelques crues se produisent entre le $15 / 7$ et le 20 septembre sans écoulement entre.

En zone sahélienne, les phénomènes d'écoulement s'étalent sur 3 mois, les autres mois le cours d'eau restant à sec. Durant ces dernières années, celles qui sont concernées par la sécheresse, les apports des cours d'eau origi- naires de la zone sahélienne ne sont connus de façon quantitative que du nord-est de la Haute-Volta à la République du Tchad, où des observations régulières ont été faites. Encore ne s'agit-il pas des régions où la sécheresse a sévi avec le plus de rigueur.

\subsection{Fleuves tropicaux parvenant au Sahel}

Il s'agit essentiellement du Sénégal, du Niger, du Logone et du Chari, qui ont un bassin supérieur bien arrosé et qui coulent vers le nord. Leur écoulement est permanent et l'hydrogramme annuel présente une seule pointe se produisant en septembre-octobre, aux stations en bordure de la zone sahélienne et un minimum en mars-avril. La traversée du Sahel conduit à des pertes énormes par évaporation dans les zones dégradées, sauf pour le Sénégal, le volume annuel pouvant se réduire de 40 à $50 \%$ dans les plaines d'inondation du Niger. L'hydrogramme annuel tend à prendre une forme plus arrondie et le maximum de crue diminue au fur et à mesure que le fleuve pénètre plus en amont dans le Sahel. En années très humides, les pertes augmentent très fortement dans le Sahel, le flot d'inondation s'étalant sur une plus grande largeur dans les plaines.

Sur ces grands fleuves qui atteignent ou traversent la bande sahélienne, l'information hydrologique est plus abondante: on dispose, en effet, de données sûres et de longues séquences d'écoulement, quelques stations étant suivies depuis le début du siècle. D’autre part, pour la période récente, les mesures effectuées par les brigades hydrologiques, en collaboration avec l'ORSTOM, permettent de connaître ou d'évaluer de façon très précise les débits de basses et de très basses eaux : les étiages observés durant la période de sécheresse sont très souvent les plus faibles depuis l'installation des stations.

\section{Données pluviométriques sur la séche- resse récente et comparaison avec les autres sécheresses connues}

\subsection{La sécheresse actuelle}

On fait débuter en général la récente période de sécheresse en 1968 car la pluviométrie de l'année 1967 a été très largement excédentaire. Toutefois, dès 1965, de vastes régions du. Mali au Tchad, au nord de l'isohyete $500 \mathrm{~mm}$, ont subi un fort déficit pluviométrique se répétant l'année suivante ; c'est ainsi que la station de Tombouctou-Kabara, au Mali, ne recueille en 1966 que $89 \mathrm{~mm}$ de pluie, record absolu, contre $210 \mathrm{~mm}$ en année médiane. Les régions au sud de l'isohyète $500 \mathrm{~mm}$ sont également touchées puisque le fleuve Niger présente pour ces deux années-là des modules voisins ou inférieurs à ceux de 1968 et qu'à N'Djamena, le Chari a, en 1965, un module inférieur à tous ceux de la période 68 à 71 .

L'évolution de la sécheresse est la suivante :

Sécheresse brutale en 1968 affectant en premier lieu la Mauritanie et le Sénégal ou la pluviométrie a souvent un temps de récurrence compris entre 10 et 20 ans.

- Année 1969 presque normale, sauf sur les parties subdésertiques du Niger et du Tchad. 
- Reprise accentuée du déficit pluviométrique en 1970 dans les pays déjà cités, mais forte irrégularité spatialc des précipitations, certaines zones de la Mauritanie. par exemple, restant excédentaires.

- Aggravation de la sécheresse en 1971 dans tous les pays du Sahel sauf au Sénégal qui est relativement épargné.

- Point culminant de la sécheresse en 1972 : tous les postes pluviométriques de la zone sahélienne sont déficitaires et l'on enregistre de nombreux records absolus, particulièrement du Sénégal au Mali. C'est ainsi que Dakar ne reçoit que $117 \mathrm{~mm}$ de pluie pour une médiane de $500 \mathrm{~mm}$.

Par bandes pluviométriques, la situation est la suivante pour cette année là

- Bande inférieure à $100 \mathrm{~mm}$ : dans la zone désertique où il n'est pas rare que les précipitations annuelles soient nulles (cas de Fada au Tchad), les données des très rares stations existantes ne sont pas d'un grand secours. On sait simplement de façon qualitative que l'année 1972 a été très sèche.

- Bande comprise en 100 et $300 \mathrm{~mm}$ : au sud de la bande désertique, la densité des stations est suffisante pour que l'on puisse arriver à une impression générale. Depuis le Soudan jusqu'à l'Atlantique, l'année 1972 a été extrêmement sèche sur cette bande limitée au sud par une ligne qui passe un peu au nord du fleuve Sénégal, à l'ouest, et qui coupe le lac Tchad à l'est. Dans cette région, quelques données tendaient à prouver que tous les 30 ou 50 ans les précipitations annuelles descendaient jusqu'à des valeurs comprises entre 30 et $60 \mathrm{~mm}$ pour des moyennes de longue durée de 200 à $300 \mathrm{~mm}$. Ceci a été largement confirmé puisque, dans la zone en question, ont été observées en 1972 un bon nombro de hauteurs annuelles comprises entre 45 et $80 \mathrm{~mm}$ (déficit de l'ordre de $75 \%$ ). Pour de telles hauteurs, les phénomènes de ruissellement deviennent très rares. Il est vraisemblable que dans cette zone le temps de récurrence est compris entre 50 et 100 ans

- Bande comprise entre 300 et $750 \mathrm{~mm}$ : au sud de la zone précédente, l'agriculture intéresse des surfaces beaucoup plus importantes et connait, en année normale, des conditions beaucoup moins précaires; le déficit en valeur relative parait nettement moins élevé mais les conséquences pratiques ont été tout aussi graves : entre les isohyètes 300 et $400 \mathrm{~mm}$, le déficit varie généralement de 60 à $50 \%$; enfin, plus au sud. il varie entre 40 et $25 \%$. Le temps de récurrence varie entre 10 et 50 ans.
Bien que nous nous soyons limités au Sahel, l'extension de la sécheresse est telle que la pluviométrie des régions méridionales doit également ètre évoquée :

- Bande comprise entre 750 et $1200 \mathrm{~mm}$ : Le temps de récurrence varie suivant les régions entre 10 et 20 ans, atteignant parfois 50 ans. Le Sénégal, le sud du Mali et le nord-ouest de la Haute-Volta sont très touchés, la ville de Bamako ne reçoit que $728 \mathrm{~mm}$ de pluie, record absolu, contre $1040 \mathrm{~mm}$ en année médiane.

- Bande supérieure à $1200 \mathrm{~mm}$ : le déficit pluviométrique, bien que beaucoup plus modeste et ne dépassant pas un temps de récurrence de 10 ans, se manifeste encore dans toutes les régions nord des pays côtiers. A l'est de la zone étudiće, il atteint le Cameroun et la R.C.A. jusqu'a environ $4^{\circ}$ de latitude nord, ou le temps de récurrence de l'écoulement est beaucoup plus fort (Sanaga, Oubangui, les pluies étant mal réparties dans l’année). en 1973, les précipitations sont, dans l'ensemble, un peu plus fortes dans toutes les bandes climatiques et principalement dans la zone supérieure à $1200 \mathrm{~mm}$ où, à part la région de Bougouni au Mali, la récurrence n'excède pas 5 ans. Mais certaines régions relativement épargnées en 1972 ne l'ont pas été en 1973 (en Haute-Volta et dans le sud du Mali notamment) et on retrouve pour cette demière année un nombre non négligeable de valeurs minimales absolues correspondant à des récurrences de 50 ou 100 ans pour des postes qui, en 1972, avaient présenté des fréquences nettement plus élevées. C'est le cas, entre autre, d'Abeche (Tchad) qui reçoit $188 \mathrm{~mm}$ de pluie seulement contre $420 \mathrm{~mm}$ en année médiane. La répartition des précipitations mensuelles a, par endroits, été plus favorable aux pâturages qu'en 1972.

En 1974, la pluviométrie redevient proche de la normale à peu près partout, sauf au Sénégal où, à part certaines régions excédentaires, la plupart des postes ne recueillent guère plus de précipitations qu'en 1973.

- En 1975, l'amélioration se confirme mais les précipitations et les écoulements restent très voisins des valeurs moyennes.

\subsection{Comparaison avec les autres sécheresses connues}

Comment se place cette sécheresse par rapport aux deux autres sécheresses historiques : celle de 1941 à 1945 et surtout celle de 1907 a 1914, qui semble avoir été plus rigoureuse par l'importance des souvenirs qu'en ont gardés les populations. Le tableau 1 donne, pour 8 stations

Episodes pluvieux les plus secs (moyenne annuelle en $\mathrm{mm}$ )

Tableau

\begin{tabular}{|c|c|c|c|c|c|c|c|c|c|}
\hline \multirow{2}{*}{ Station } & \multirow{2}{*}{$\begin{array}{l}\text { Total } \\
\text { annuel } \\
\text { médian }\end{array}$} & \multicolumn{8}{|c|}{ Moyenne annuelle la plus faible sur } \\
\hline & & \multicolumn{2}{|c|}{1 an } & \multicolumn{2}{|c|}{2 ans } & \multicolumn{2}{|c|}{5 ans } & \multicolumn{2}{|c|}{10 ans } \\
\hline Dakar & 500 & 117 & 1972 & 202 & $72-73$ & 263 & $70-74$ & 423 & $65-74$ \\
\hline Saint-Louis & 330 & 144 & 1914 & 147 & $13-14$ & 179 & $70-74$ & 284 & $65-74$ \\
\hline Segou & 710 & 456 & 1949 & 517 & $48-49$ & 569 & $70-74$ & 630 & $40-49$ \\
\hline Tombouctou & 210 & 89 & 1966 & 106 & $72-73$ & 137 & $70-74$ & 146 & $65-74$ \\
\hline Ouagadougou & 810 & 408 & 1913 & 505 & $12-13$ & 580 & $10-14$ & 669 & $7-16$ \\
\hline Niamey & 580 & 290 & 1915 & 319 & $14-15$ & 383 & $12-16$ & 443 & $10-19$ \\
\hline Zinder & 490 & 215 & 1912 & 222 & $12-13$ & 305 & $11-15$ & 391 & $65-74$ \\
\hline N'Djamena & 620 & 306 & 1913 & 370 & $73-74$ & 487 & $70-74$ & 525 & $65-74$ \\
\hline
\end{tabular}


existant au moins depuis 1907, mais parfois avec des lacunes importantes (Tombouctou et N'Djamena notamment), le record pluviométrique annuel absolu et la moyenne annuelle des épisodes secs les plus intenses sur 2, 5 et 10 ans. Cette comparaison très grossière car s'appuyant sur un trop petit nombre de postes, dont certains subissent déjà une influence maritime marquée, montre que:

- à l'échelle de l'année la plus faible ou de deux années consécutives, la sécheresse "1913" l'emporte largement sur la sécheresse "1972", la sécheresse "1940" étant, elle, beaucoup moins sćvère et n'apparaissant même pas dans le tableau. Les valeurs sont particulièrement basses pour Saint-Louis, Ouagadougou, Niamey et Zinder).

-à l'échelle de 5 années consécutives, la période 1970 1974 devient la plus sèche, sauf en Haute-Volta et au Niger, et cette tendance à une sévérité accrue se confirme sur 10 ans consécutifs, sauf pour Ougadougou et Niamey qui ont été relativement épargnés.

\section{L'écoulement au cours de la séche- resse récente}

\subsection{Cours d'eau sahéliens}

Les seules données disponibles concernent des cours d'eau situés au sud de l'isohyète $400 \mathrm{~mm}$ et à l'est du méridien de Greenwich donc dans les régions qui n'ont pas été les plus affectées par la sécheresse (fig. 2).

On trouve de la Haute-Volta vers le Tchad:

- les rivières situées dans la boucle du Niger, affluents de la rive droite de ce fleuve ; citons le Gorouol, le Dargol, la Sirba, les rivières situées le long de la frontière Niger-Nigéria, telles la Maggia, le Goulbi de Maradi, le Komadougou,

- les rivières de l'est du Tchad : le Bahr Azoum, cours supérieur du Bahr Salamat et le Batha.

Ces cours d'eau concernent des superficies allant de quelques milliers à quelques dizaines de milliers de $\mathrm{km}^{2}$ où la dégradation hydrologique est très variable. Ils ont dans le meilleur des cas une vingtaine d'années d'observations seulement.

L'étude de la distribution statistique des échantillons de modules annuels et des déficits annuels de 1968 à 1973 souligne bien la variabilité spatio-temporelle de l'écoulement évoquée précédemment (fig. 3).

Pour les cours d'eau sans grandes réserves scuterraines, la succession de plusieurs années sèches n'a pas d'effet cumulatif et le déficit dépend de façon très lâche du total pluviométrique annuel. La répartition des précipitations au cours de la saison des pluies a un caractere prédominant sur l'écoulement et l'hétérogénéité spatiale de celui-ci est considérable. En 1972, dans l'extrémité ouest du Niger, et pour des totaux pluviométriques voisins, l'écoulement du Dargol $\left(2750 \mathrm{~km}^{2}\right)$ est proche de la valeur moyenne, par contre la Sirba $\left(38750 \mathrm{~km}^{2}\right)$ accuse un deficit de $70 \%$. C'est pour cette raison que la grande sécheresse des années 68 et 72 ne s'est pas systématiquement traduite par des déficits records au point de vue hydrologique et l'on a mème pu observer localement, en Haute-Volta notamment, des crues importantes.

- Les cours d'eau dont le régime hydrologique d'une année est lié à l'abondance de l'année précédente, c'est-à-dire ceux qui traversent de vastes zones plus ou moins marécageuses, comme la Komadougou, ou qui sont alimentés par de grandes nappes souterraines

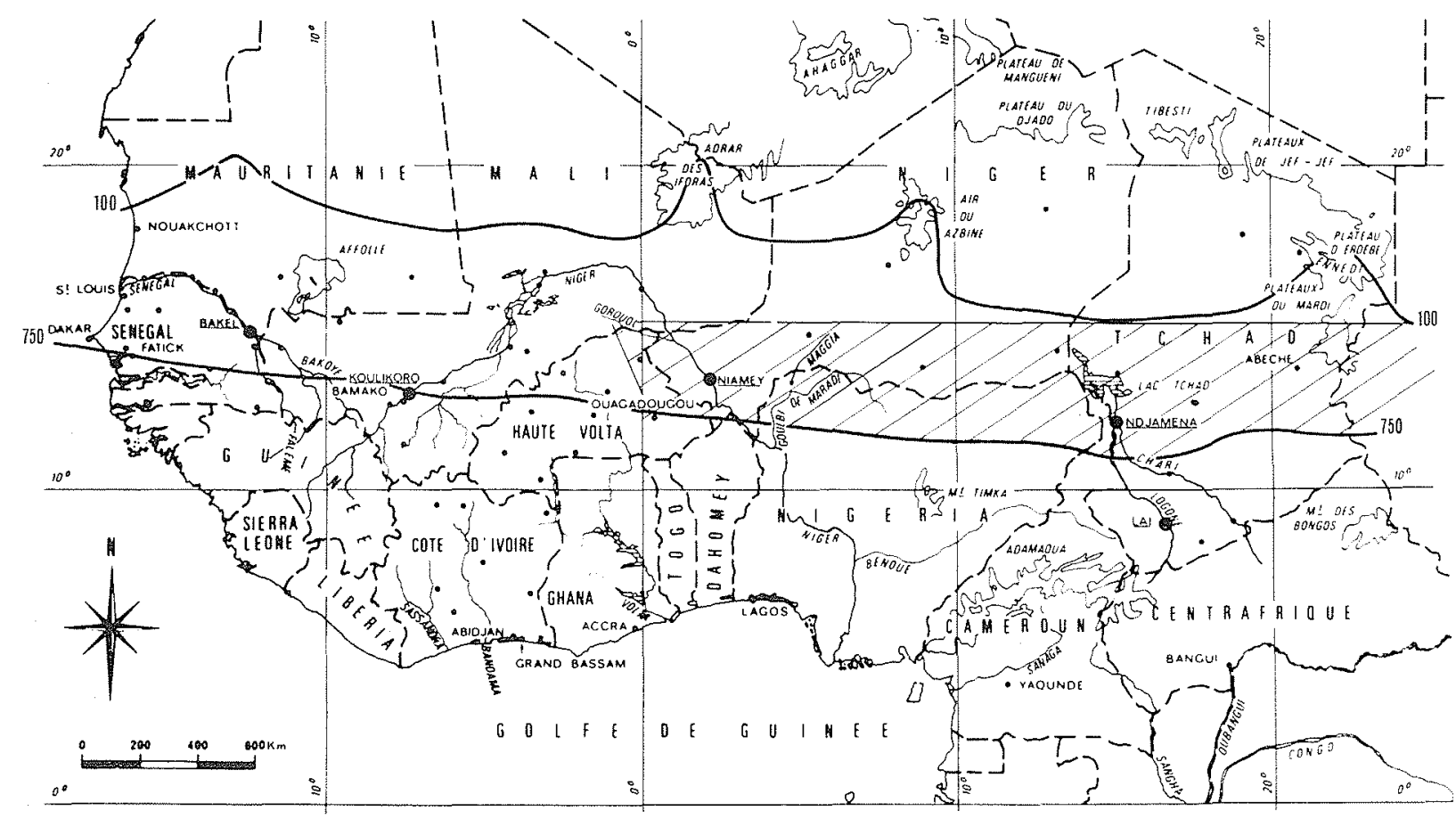

2/ Cours sahéliens suivis au cours de la sécheresse 1968-1973. 


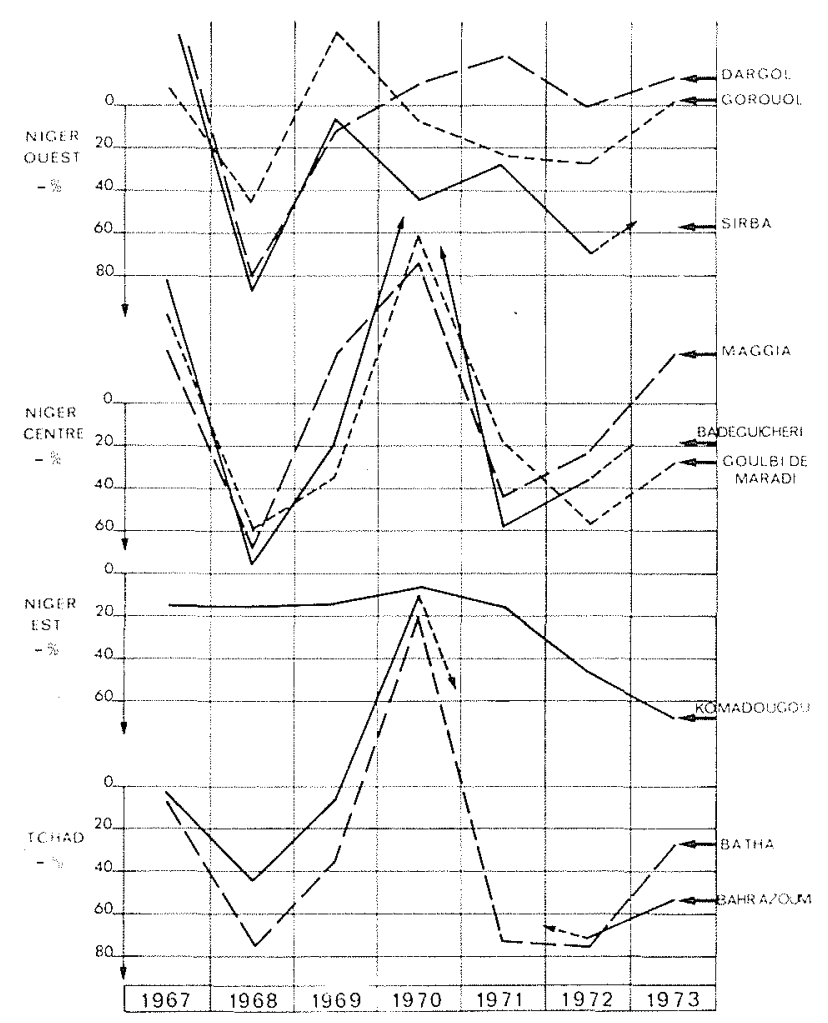

3/ Evolution en \% du déficit ou excédent annuel - Cours d'eau sahéliens

à faible profondeur, comme la Korama au sud de Zinder (Niger), présentent, à la fin de la période, des déficits beaucoup plus graves : la récurrence du module 1973 de la Komadougou est de 40 ans, avec un écoulement égal au tiers de l'écoulement médian. Sur la Korama, dont l'écoulement annuel a été nul en 1973, la récurrence est d'au moins 20 ans, peut-être 40 ans, alors que l'année sèche 1968 isclée n'a pas eu de conséquences aussi graves.

- D’une façon générale, le caractère déficitaire de chaque année a donc varié largement d'un cours d'eau à l'autre sur cette partie de la bande sahélienne et l'année la plus déficitaire n'a pas été la même partout. L'année 1972 présente des écoulements très réduits partout, mais ce n'est qu'au Tchad qu'elle atteint des records. Le déficit de la Komadougou, qui se jette dans le lac Tchad, atteint son maximum en 1973 ; dans les régions de l'ouest et du centre du Niger, c'est l'année 1968 qui est largement la plus sèche avec un déficit atteignant parfois $80 \%$ de l'écoulement en année moyenne.

- Pour spectaculaire qu'il soit, le déficit atteint en 1968 ou en 1972 n'est pas obligatoirement d'une grande rareté, les cours d'eau sahéliens ayant souvent une irrérularité interannuelle très forte. C'est ainsi que, malgré la brièveté des périodes d'observations (15 à 20 ans), la récurrence de l'année la plus déficitaire ne parait pas être très élevée. Du Mali au lac Tchad, elle semble être comprise entre 5 et 15 ans, atteignant parfois 25 ans lorsque c'est l'année 68 qui est la plus faible. Au Tchad, la sécheresse a été plus sévère et les temps de récurrence pour les grands cours d'eau varient de 10 à 20 ans. Du Sénégal à l'ouest de la Haute-Volta où l'on ne dispose malheureusement pas d'observation, il semble toutefois que la rareté du phénomène soit beaucoup plus forte et que le temps de récurrence soit compris entre 50 et 100 ans.

\subsection{Grands fleuves tropicaux parvenant au Sahel}

Ces fleuves sont caractérisés par un déficit relatif beaucoup moins importart que celui des cours d'eau sahéliens, mais la fréquence d'occurrence de la sécheresse y est beaucoup plus faible. Dans la partic amont de ces cours d'eau, à leur arrivée dans le Sahel, où l'eau est relativement abondante, ce déficit a eu des conséquences beaucoup moins dramatiques qu'au Sahel, malgré la plus grande rareté des phénomènes observés. Dans leurs cours inférieurs, il n'en est pas du tout de même; soit parce que les crues sont insuffisantes pour envahir les plaines d'inondation où se pratiquent les cultures de décrues (cas notamment du Sénégal), soit parce que les pertes (bien qu'inférieures en volume à celles des années humides) amputent lors de la traversée du Sahel environ $40 \%$ d'un module déjà modeste (c'est le cas du Niger à sa sortie de la cuvette lacustre, dont le module 73 s'abaisse à $560 \mathrm{~m}^{3} / \mathrm{s}$ à Dire, alors qu'il était de $903 \mathrm{~m}^{3} / \mathrm{s}$ à Koulikoro).

Au niveau des modules annuels on constate que : (fig. 4)

- l'année 1968 n'est fortement déficitaire que pour le fleuve Sénégal uniquement $(-45 \%$, temps de récurrence 17 ans);

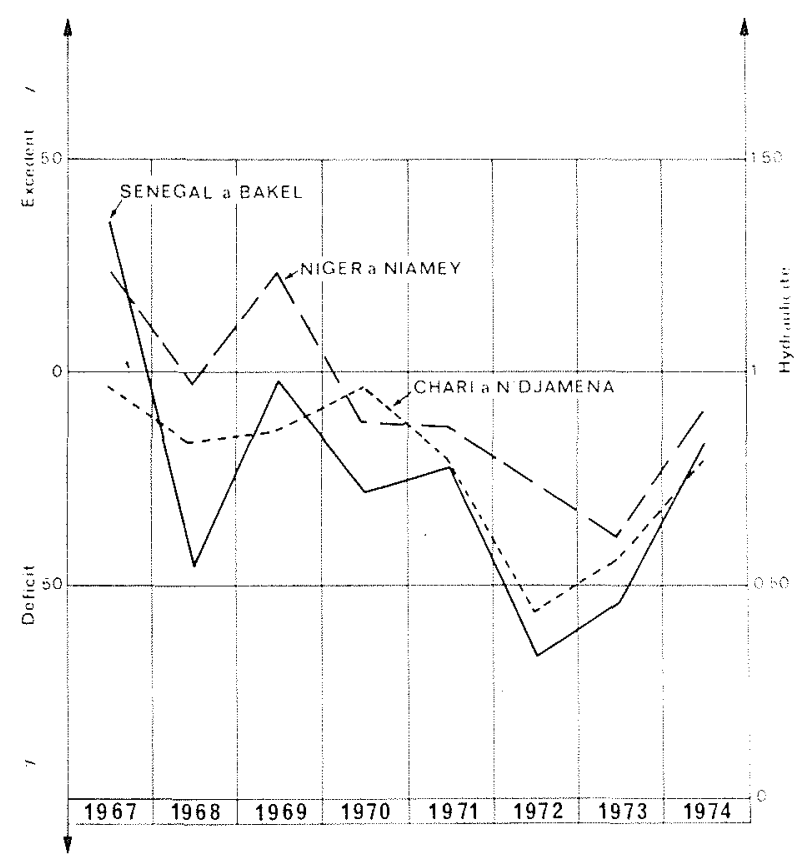

4/ Evolution du déficit annuel (en \%) des grands fleuves parvenant au Sahel

- l'année 1969 est très excédentaire pour le fleuve Niger, normale pour le Sénégal, par contre le fleuve Chari a un déficit de $14 \%$;

- on note ensuite une succession d'années systématiquement déficitaires de 1970 à 1973, les deux années les plus faibles étant 1972 et 1973 ;

- pour les fleuves Sénégal, Chari et Logone, les plus faibles modules de toute la période de mesure s'observent en 1972 et le temps de récurrence est compris entre 50 et 100 ans, le déficit atteint $66 \%$ à Bakel et $56 \%$ à N'Djamena , (fig. 5, 6); 


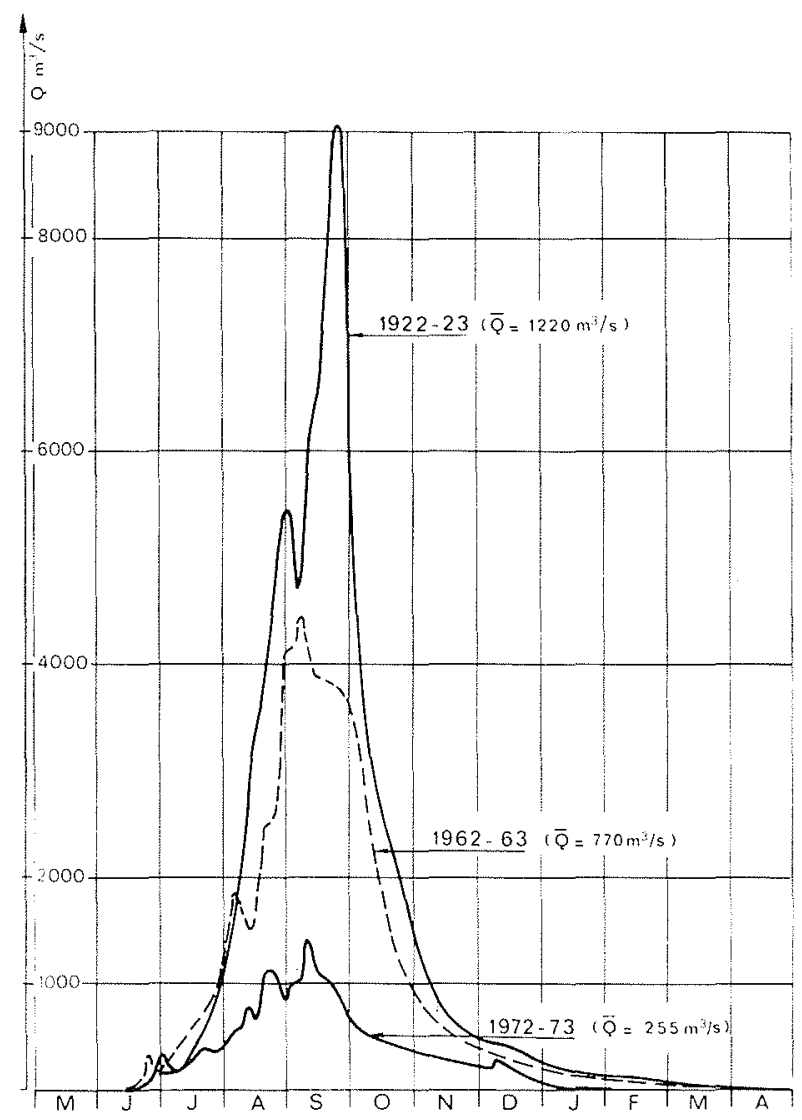

5/ Le Sénégal à Bakel - Hydrogrammes annuels

- pour le fleuve Niger, c'est l'année 1973 qui est la plus mauvaise avec une récurrence voisine de 50 ans, mais l'année 1913 (connue seulement à Koulikoro) était était encore plus faible. Cependant, à l'aval de la cuvette lacustre, il est fort possible que la fréquence ait été plus faible, les déficits ayant tendance à être plus élevés qu'à l'amont, (fig. 7,8).

Les débits maximaux de crue ne dépassent pas, au cours de cette sécheresse, des valeurs très modestes et le temps de récurrence est de l'ordre de 50 ans pour ces grands fleuves. Les plus faibles valeurs observées se produisent en 1972, mais restent légèrement au-dessus de celle de l'année 1913.

- On note ainsi pour le Sénégal à Bakel : $1430 \mathrm{~m}^{3} / \mathrm{s}$ en 1972 pour $1040 \mathrm{~m}^{3} / \mathrm{s}$ en 1913 .

- Sur le fleuve Niger, la crue atteint à Koulikoro : $3680 \mathrm{~m}^{3} / \mathrm{s}$ en 1972 contre $3450 \mathrm{~m}^{3} / \mathrm{s}$ en 1913

- A N'Djamena, la pointe annuelle du Chari s'abaisse à $1430 \mathrm{~m}^{3} / \mathrm{s}$ en 1972 , plus faible valeur depuis le début des mesures en 1932 .

Les étiages sont excessivement rigoureux et les plus faibles valeurs de la période 1968 à 1974 s'observent en 1974 à la fin de l'année hydrologique 1973, l'effet cumulatif de la recharge insuffisante des nappes pendant plusieurs années consécutives se faisant très nettement sentir.

On observe des valeurs très basses, voire nulles :

- assèchement total du Sénégal à Bakel pendant huit jours consécutifs au mois d'avril;

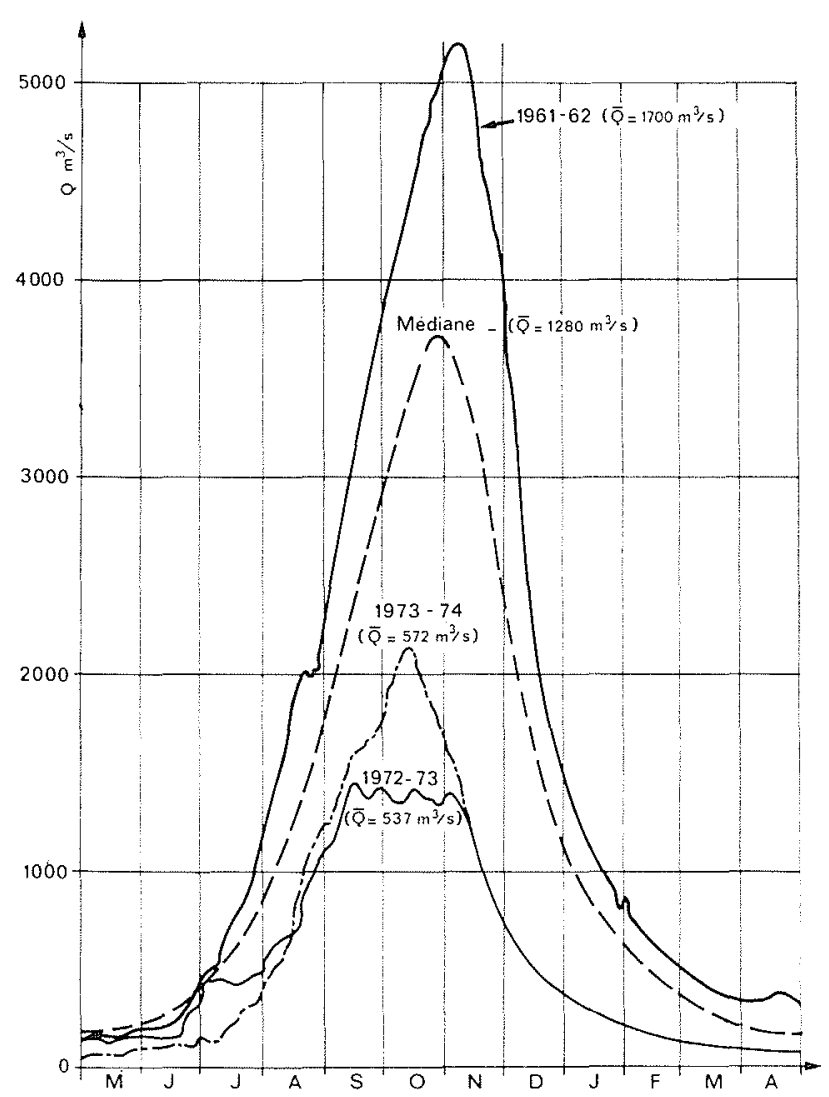

6/Le Chari à N'Djamena (Fort-Lamy) - Hydrogrammes annuels

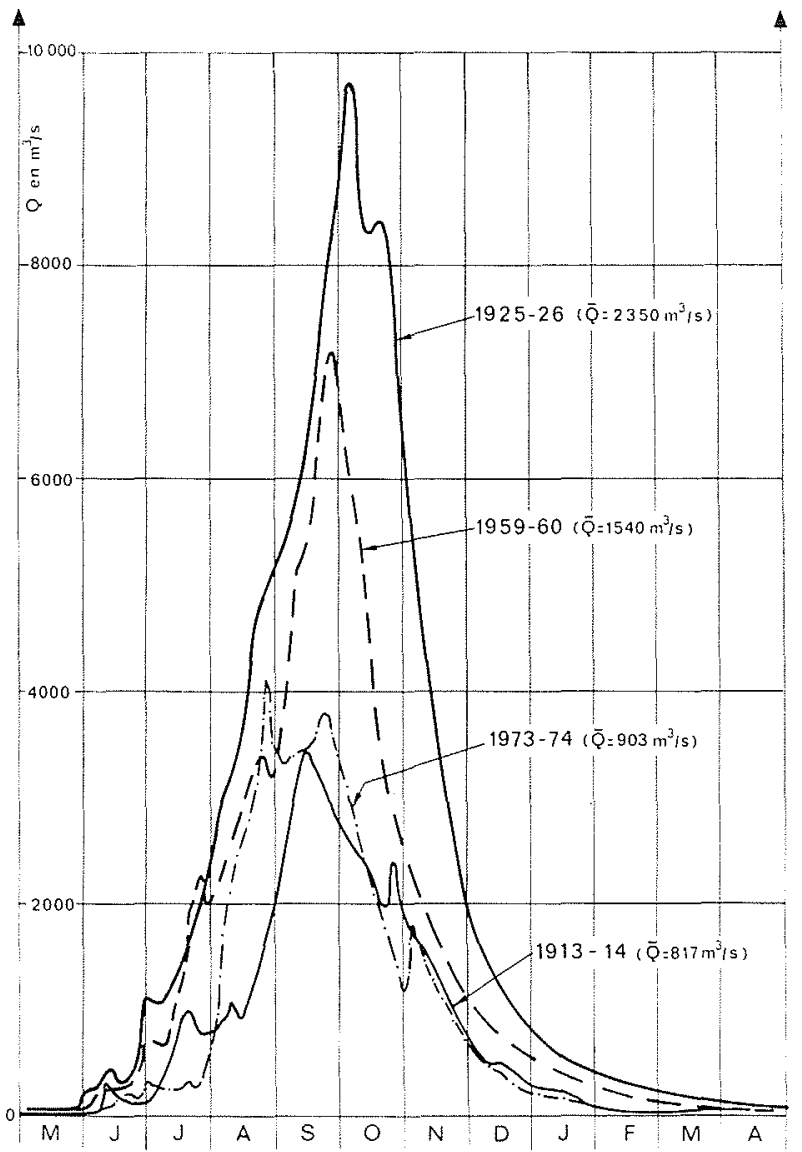

$7 /$ Le Niger à Koulikoro - Hydrogrammes annuals 


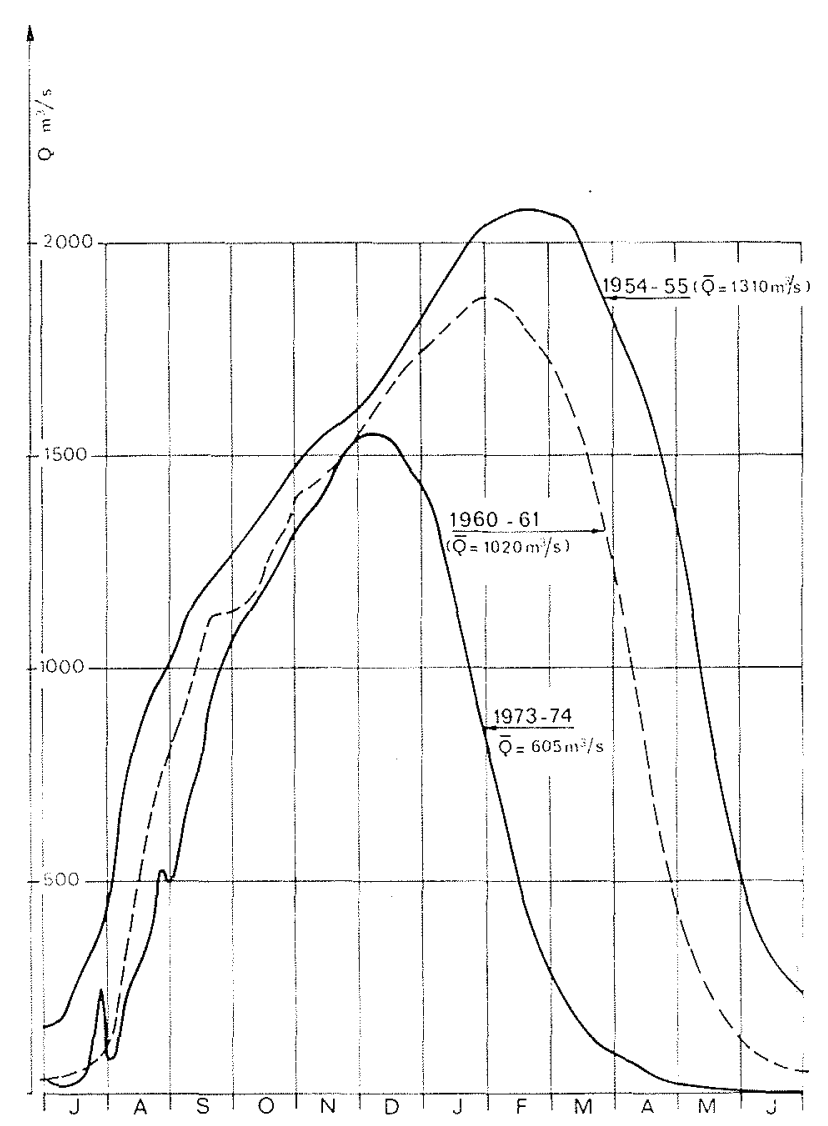

8/ Le Niger à Niamey - Hydrogrammes annuels sur le Niger, le débit d'étiage s'abaisse à $0,6 \mathrm{~m}^{3} / \mathrm{s}$ à Niamey au mois de juillet, alors que la valeur de l'étiage moyen est de $75 \mathrm{~m}^{3} / \mathrm{s}$.

\section{Comparaison avec les autres séche- resses connues}

Il n'existe aucune donnée hydrologique concernant les cours d'eau sahéliens sur la sécheresse 1940-1945 et a fortiori sur la sécheresse 1913-1914. Cependant, malgré la brièveté de la période d'observation sur les cours d'eau suivis de l'est de la Haute-Volta au Tchad, il ne semble pas, au moins pour cette zone, que la récurrence soit inférieure à cinquante ans puisque dans bon nombre de cas où les observations sont completes depuis vingt ans, on observe des années, telles 1957 ou 1960, plus déficitaires que 1968 on 1972.

En ce qui concerne les grands fleuves, les observations continues faites à Bakel sur le Sénégal depuis 1903, à Koulikoro sur le Niger depuis 1907 et à N'Djamena sur le Chari depuis 1932 (jl manque donc, pour le Chari,les relevés sur la sécheresse du début du siècle) permettent des constatations intéressantes (cf. tableau 2 et fig. 9, 10.11).

- A l'échelle annuelle, 1972 ou 1973 semblent avoir été des records absolus au plan des apports annuels depuis le début du siècle (sauf sur la partie du Niger en amont de la cuvette lacustre) et peut-être également en ce qui concerne les étiages absolus, ceux-ci n'étant connus avec précision que par la sécheresse récente. Toutefois

\begin{tabular}{|c|c|c|c|c|c|c|c|}
\hline & & & & & & & Tableau 2 \\
\hline $\begin{array}{l}\text { Station et } \\
\text { période } \\
\text { d'observation }\end{array}$ & $\begin{array}{c}\text { Débit moyen } \\
\text { annuel } \\
\mathrm{m}^{3} / \mathrm{s}\end{array}$ & \multicolumn{2}{|c|}{$\begin{array}{l}\text { Récurrence } \\
\text { Rang }\end{array}$} & $\begin{array}{l}\text { Débit maximal } \\
\qquad \mathrm{m} 3 / \mathrm{s}\end{array}$ & \multicolumn{2}{|c|}{$\begin{array}{l}\text { Récurrence } \\
\text { Rang }\end{array}$} & $\begin{array}{l}\text { Débit minimal } \\
\qquad \mathrm{m}^{3} / \mathrm{s}\end{array}$ \\
\hline \multirow[b]{2}{*}{$\begin{array}{l}\text { Sénégal à } \\
\text { Bakel } \\
1903-1974\end{array}$} & (moyenne: $/ 10)$ & & & $($ moyenne : 4720$)$ & \multirow[b]{2}{*}{$\begin{array}{l}1 \\
4 \\
3 \\
2 \\
6\end{array}$} & \multirow[b]{2}{*}{$\begin{array}{r}100 \\
20 \\
30 \\
50 \\
15\end{array}$} & (mediane : 2,3 ) \\
\hline & $\begin{array}{l}1913=272 \\
1914=444 \\
1944=331 \\
1972=255 \\
1973=355\end{array}$ & $\begin{array}{r}2 \\
10 \\
3 \\
1 \\
4\end{array}$ & $\begin{array}{r}50 \\
12 \\
25 \\
100 \\
20\end{array}$ & $\begin{array}{l}1913=1040 \\
1914=1880 \\
1944=1740 \\
1972=1430 \\
1973=2250\end{array}$ & & & $\begin{array}{l}1973=0,25 \\
1974=0\end{array}$ \\
\hline \multirow[b]{2}{*}{$\begin{array}{c}\text { Niger à } \\
\text { Koulikoro } \\
1907-1974\end{array}$} & (moyenne : 1540 ) & & & (moyenne: 6140 ) & \multirow[b]{2}{*}{$\begin{array}{l}1 \\
6 \\
3 \\
7 \\
2 \\
4\end{array}$} & \multirow[b]{2}{*}{$\begin{array}{r}100 \\
15 \\
8 \\
8 \\
50 \\
20\end{array}$} & (médiane $=45$ ) \\
\hline & $\begin{array}{l}1913=817 \\
1914=929 \\
1942=993 \\
1944=993 \\
1972=1080 \\
1973=903\end{array}$ & $\begin{array}{l}1 \\
3 \\
5 \\
5 \\
7 \\
2\end{array}$ & $\begin{array}{r}100 \\
30 \\
15 \\
15 \\
10 \\
50\end{array}$ & $\begin{array}{l}1913=3450 \\
1914=4470 \\
1940=4010 \\
1942=4920 \\
1972=3680 \\
1973=4140\end{array}$ & & & $\begin{array}{l}1913=(19) \\
1915=(21) \\
1944=(25) \\
1945=(19) \\
1973=16,0 \\
1974=17,4\end{array}$ \\
\hline \multirow[b]{2}{*}{$\begin{array}{c}\text { Chari à } \\
\text { N'Djamena } \\
1932-1974\end{array}$} & (moyenne : 1230 ) & & & (moyenne: 3560 ) & & & (médiane : 105) \\
\hline & $\begin{array}{l}1913=? \\
1914=? \\
1940=805 \\
1941=739 \\
1972=537 \\
1973=572\end{array}$ & $\begin{array}{l}4 \\
3 \\
1 \\
2\end{array}$ & $\begin{array}{c}10 \\
15 \\
50-100 \\
50\end{array}$ & $\begin{array}{l}1913=? \\
1914=? \\
1940=2260 \\
1941=2190 \\
1972=1430 \\
1973=2130\end{array}$ & $\begin{array}{l}4 \\
3 \\
1 \\
2\end{array}$ & $\begin{array}{c}25 \\
30 \\
50-100 \\
50\end{array}$ & $\begin{array}{l}1913=? \\
1914=? \\
1940=(80) \\
1941=(79) \\
1973=47,7 \\
1974=38,6\end{array}$ \\
\hline
\end{tabular}



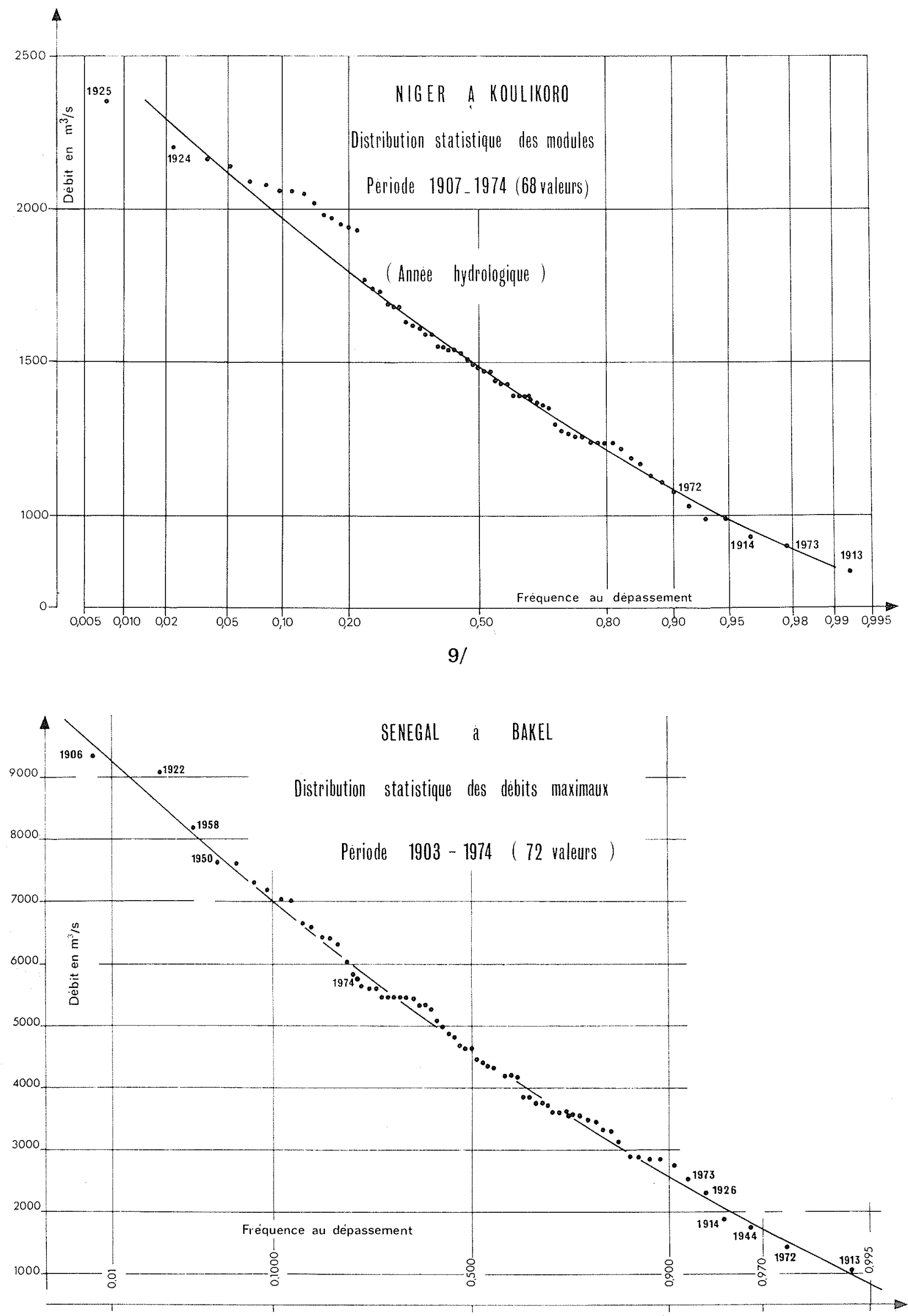


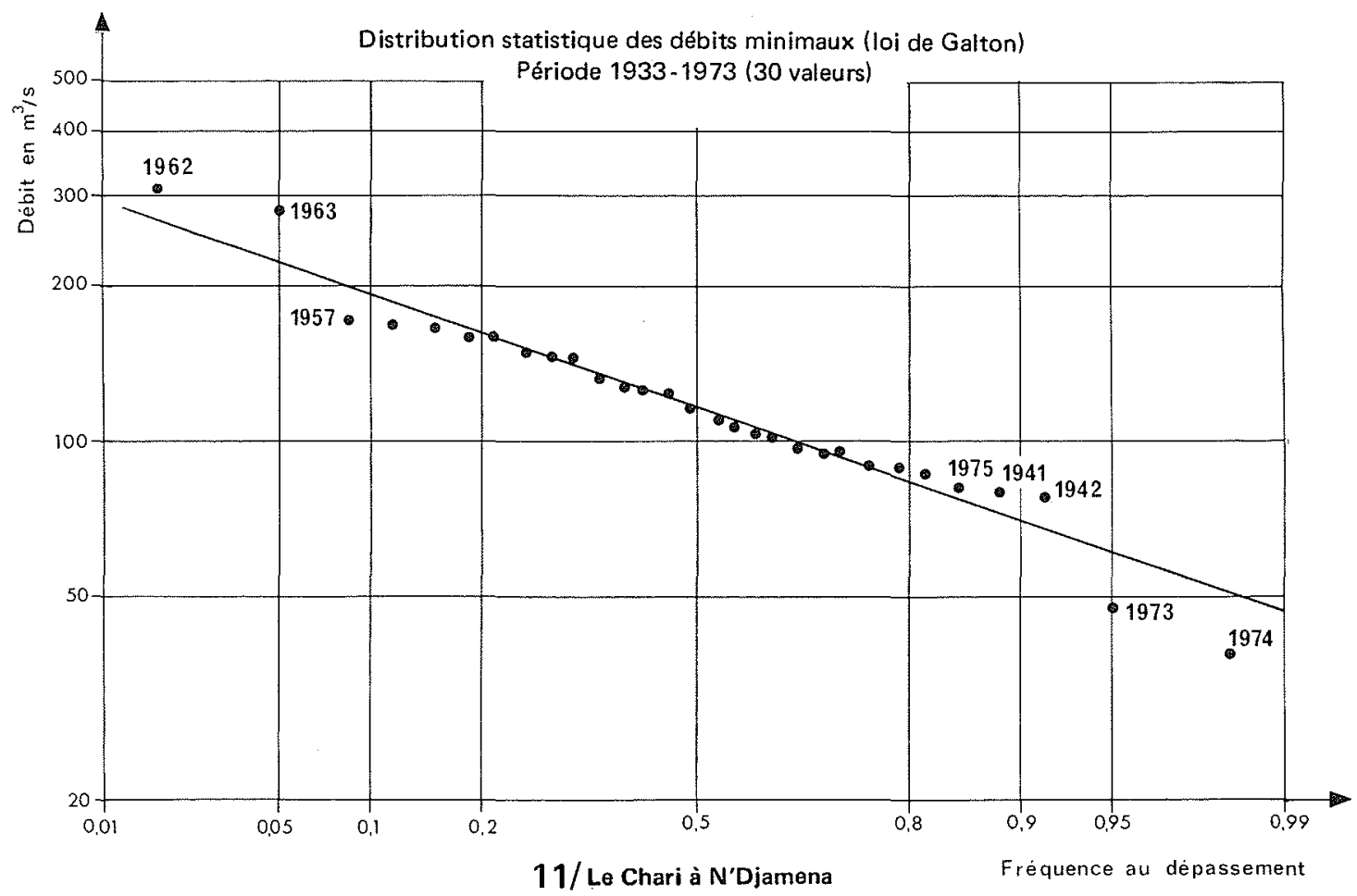

les estimations faites sur le Niger montrent que les basses eaux $\left({ }^{2}\right)$ devaient être assez voisines des valeurs observées ces dernières années et les traditions orales rapportent que le Sénégal s'est déjà asséché au cours de l'époque historique. Par contre, les débits maximaux de crue de 1972 ont été un peu supérieurs à ceux de 1913, venant se classer tout de même au 2 ème rang.

- A l'échelle de 3 ou de 5 ans (tableau 3), la dernière sécheresse semble être pour les grands fleuves tropicaux à peu près équivalente à celles des années 1910 à 1914 ou 1940 à 1944.

\section{Evolution du lac Tchad}

\subsection{Etat actuel du lac Tchad}

Le lac Tchad, constitué par deux cuvettes séparées par une zone de hauts fonds (la Grande Barrière), est une nappe d'eau endoréique peu profonde, ce qui le rend particulièrement vulnérable aux atteintes de la sécheresse. Il réagit fidelement aux variations du climat et constitue donc un indicateur précieux de la situation actuelle. Etroitement dépendant des apports du fleuve Chari qui foumissent plus de $80 \%$ de son alimentation, il présente une fluctuation: annuelle qui suit avec un certain décalage les variations de l'hydrogramme de ce fleuve : le niveau du lac connaît ainsi chaque année un minimum en juillet et un maximum en décembre-janvier (fig. 12)

Après une brève montée de son plan d'eau de 1953 à 1963, le lac connaît depuis 1964 une baisse continue qui s'est aggravée depuis 1968, les apports ayant tous été déficitaires de 1965 à 1974.

Si l'on prend comme station de référence la station de Bol, suivie depuis 1956, on peut évaluer, en janvier 1963, la surface du lac à $23500 \mathrm{~km}^{2}$ et le volume des eaux stockées à $110.10^{7} \mathrm{~m}^{3}$ pour une cote moyenne un peu supérieure à $283 \mathrm{~m}$. A partir de 1964, le plan d'eau commence à baisser d'environ $0,30 \mathrm{~m}$ entre maximums annuels successifs. Après la crue de 1967, la baisse s'amplifie, les apports de 1968 à 1971 présentant un déficit

\begin{tabular}{|c|c|c|c|c|c|c|}
\hline \multicolumn{6}{|c|}{ Modules moyens (en $\mathrm{m}^{3} / \mathrm{s}$ ) sur 3 ans consécutifs } & Tableau 3 \\
\hline & Module & Période & Module & Période & Module & Période \\
\hline $\begin{array}{l}\text { Sénégal à Bakel } \\
\text { Niger à Koulikoro }\end{array}$ & $\begin{array}{r}401 \\
1015\end{array}$ & $\begin{array}{l}71-73 \\
12-14\end{array}$ & $\begin{array}{r}427 \\
1060\end{array}$ & $\begin{array}{l}40-42 \\
42-44\end{array}$ & $\begin{array}{r}435 \\
1084\end{array}$ & $\begin{array}{l}12-14 \\
71-73\end{array}$ \\
\hline \multicolumn{7}{|c|}{ Modules moyens (en $\mathrm{m}^{3} / \mathrm{s}$ ) sur 5 ans consécutifs } \\
\hline & Module & Période & Module & Période & Module & Période \\
\hline $\begin{array}{l}\text { Sénégal à Bakel } \\
\text { Niger à Koulikoro }\end{array}$ & $\begin{array}{r}455 \\
1120\end{array}$ & $\begin{array}{l}40-44 \\
40-44\end{array}$ & $\begin{array}{r}476 \\
1150\end{array}$ & $\begin{array}{l}70-74 \\
11-15\end{array}$ & $\begin{array}{r}486 \\
1150\end{array}$ & $\begin{array}{l}10-14 \\
70-74\end{array}$ \\
\hline
\end{tabular}

(2) "1913" 


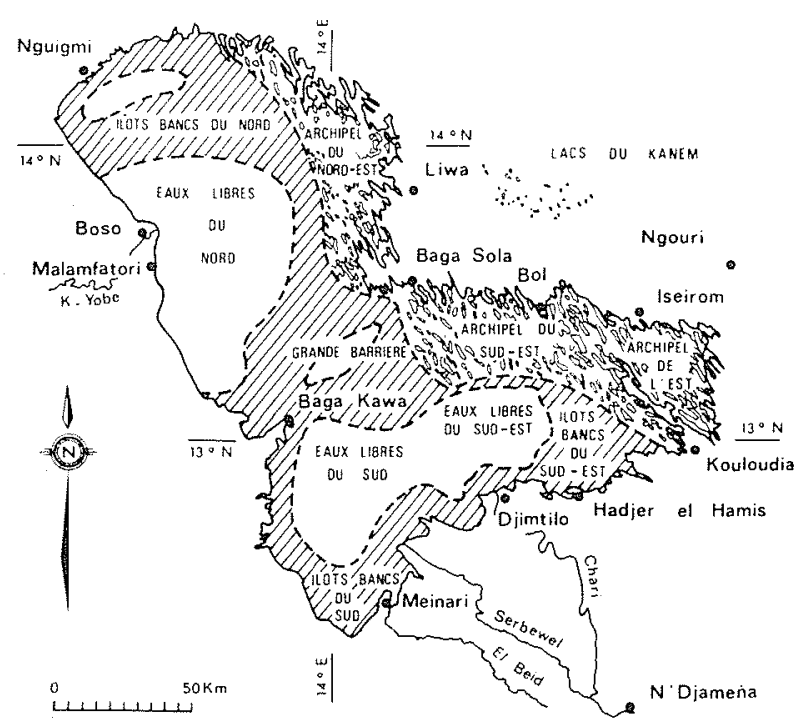

12/ Le lac Tchad à la cote 281,50 (minimum en période de pluviosité moyenne)

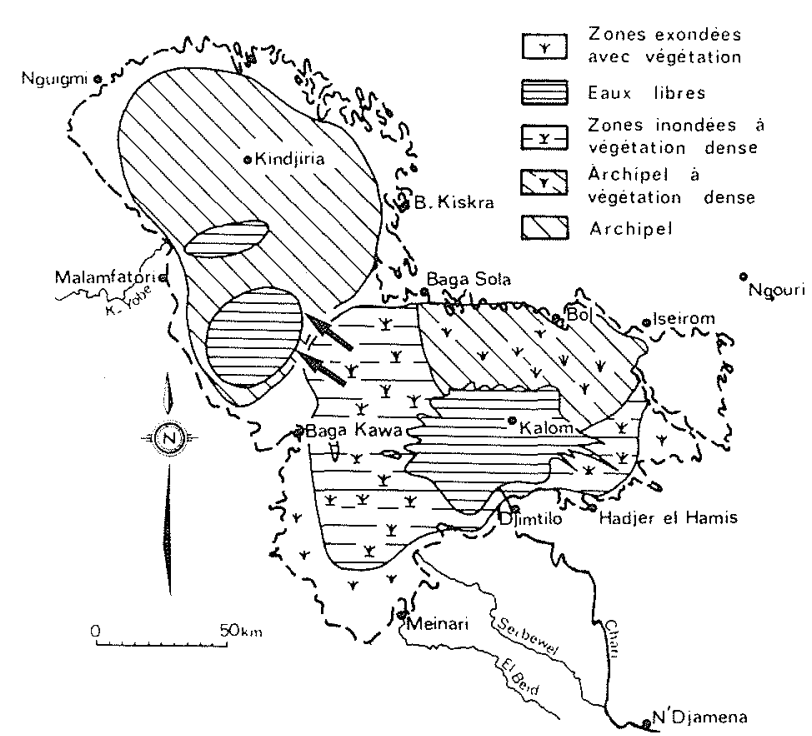

13/ Le Lac Tchad fin 1973 - début 1974 (niveau maximum 1973-1974)

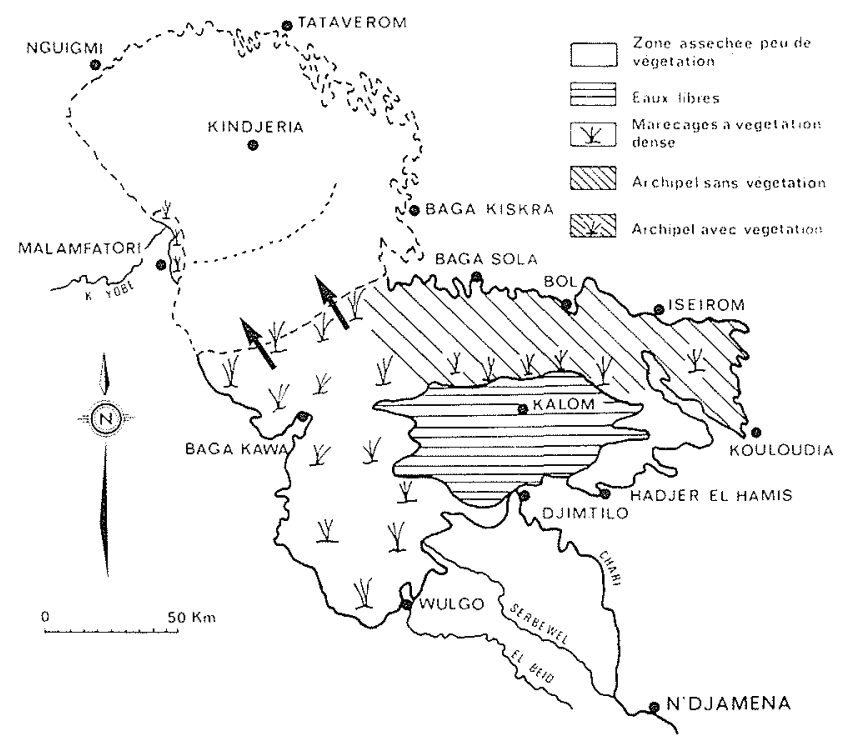

14/ Le Lac Tchad en novembre 1975

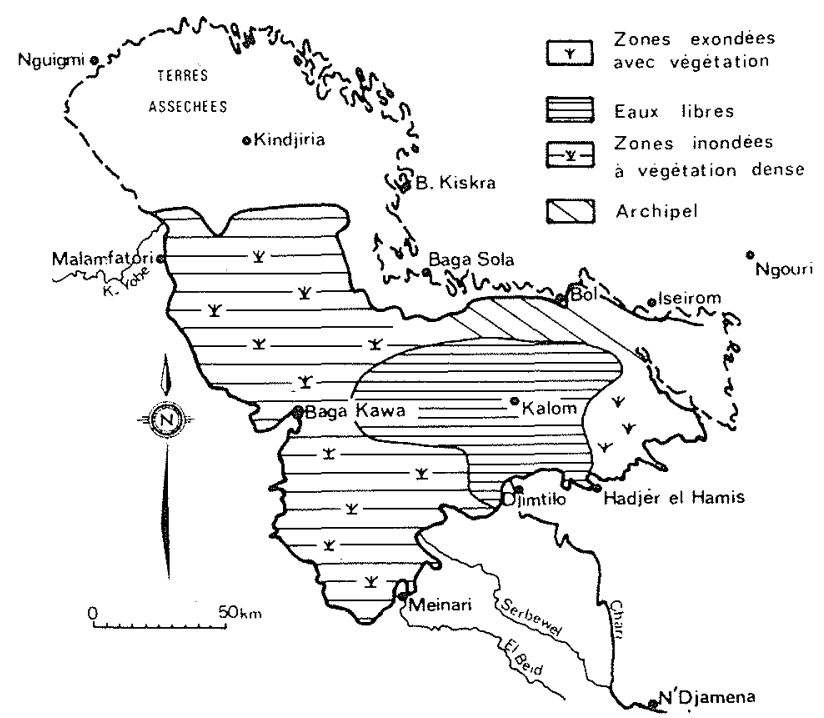

15/ Le Lac Tchad au début de 1908 (d'après le Général Tilho) niveau maximum 1907 -1908) 


\section{Conclusion}

Nous nous sommes volontairement limités à la partie descriptive des paramètres quantitatifs précipitation et écoulement des sécheresses de la période historique récente pour laquelle on dispose de données d'observations.

Les aspects hydrologiques de la dernière sécheresse, dont la sévérité n'a diminué que depuis 1974 , sont connus de façon satisfaisante pour les grands fleuves tropicaux pour lesquels la récurrence du phénomène est de 50 ans pour le Niger et est comprise entre 50 et 100 ans pour le Sénégal et le Chari. La sécheresse des cours d'eau sahéliens observés de la Haute-Volta au Tchad a un temps de récurrence plus faible, en général, de 20 ans mais atteignant localement 40 ans. De la Mauritanie au Sénégal, la sécheresse, plus sévère, doit dépasser une récurrence de 50 ans. A l'est de la zone, la baisse des eaux du lac Tchad et la coupure de celui-ci en deux cuvettes est un événement spectaculaire mais non rarissime puisque déjà observé dans un passé assez récent : là aussi, le temps de récurrence doit être voisin du demi-siècle.

Depuis le début du siècle, deux périodes de sécheresse, de 1905 à 1913 et de 1940 à 1945 , présentent une rigueur voisine. Si la sécheresse de "1940" semble être la moins intense des trois, il y a lieu de penser, par contre, que la sécheresse de "1913", qui a été tout aussi dévastatrice, l'emporte par certains aspects sur la sécheresse actuelle.

Le problème général de la sécheresse, par ses multiples facettes, intéresse de nombreuses disciplines; il comporte un certain nombre de questions angoissantes quant aux causes profondes de la sécheresse et aux conséquences du retour d'un tel phénomène. Il est malheureusement fort probable de revoir, quelle que soit la tendance climatique, une période de sécheresse comparable à celle de 1965 à 1973, disons tous les cinquante ans. Il appartient à tous les pays du Sahel de mener une stratégie anti-sécheresse commune et à long terme, si l'on veut éviter de revoir des effets aussi catastrophiques.

\section{Discussion}

President : M. M. CARLIl:R

M. le Président remercie vivement M. SIRCOULON pour son exposé très documenté sur la sécheresse du Sahel, sujet qui a donné lieu dans la presse à de nombreux reportages. Il ouvre ensuite la discussion:

Peut-on avoir quelques renseignements sur l'extension de la sécheresse soudanaise : bassins du Nil et du Congo ? demande M. TIX ERONT.

Les périodes déficitaires de 1913 puis de 1945, répond $M$. SIR COULON se retrouvent très nettement sur le Nil et sur le Congo.
Pour la sécheresse des années 1913 , on possède de nombreuses informations sur la pluviométrie du Soudan ; pour le Nil, on dispose de séries d'observations très longues bien que fragmentaires qui montrent assez fidèlement les mêmes périodes de sécheresse.

Sur une question de $\mathrm{M}$. le Président, M. SIR COULON confirme que, depuis 1962-1963 et jusqu'à la récente sécheresse, les valeurs de la "crue annuelle" du Niger à Niamey ont été anormalement fortes. Des études sont en cours (dans le cadre de l'opération SAPHYR) pour chercher une explication à cette apparente modification de regime du fleuve.

\section{Abstract \\ The recent drought in the Sahel regions of West Africa}

The drought which recently affected the tropical regions possessed remarkable geographical extension and severity. Particularly West Africa was concerned and the consequences of the drought were dramatic in the Sahel zone.

Perceptible in certain regions from 1965, this drought became noticeable in Mauritania, Senegal, North Mali and Niger in 1968 It became more severe in 1970 and extended to Upper Volta and Chad. Numerous rainfall stations measured absolute record low precipitation values. In many parts of the Sahel, the driest year was 1972, with precipitation occurrence frequency neighbouring the centennial value for the Sahel and humid tropical regions further south. Although rainfall returned to near-normal in 1974, the effects of the drought have hardly begun to disappear.

The runoff data obtained by hydrological services in collaboration with ORSTOM clearly reflect the extreme severity of the drought. The big Sahelian rivers carried annual flow volumes of occurrence frequencies between 0.1 and 0.05 ; seasonal precipitation distribution partly balanced the global annual deficit in many cases.
For several rivers, 1968 was drier than 1972 or 1973 . In the south, however, all the big tropical rivers - i.e. the Senegal, Niger and Chari - carried mean annual and maximum daily flows of an occurrence frequency close to 0.01 , with a deficit exceeding $50 \%$. Low flows were extremely small : lowest discharge at Niamey was $0.6 \mathrm{~m}^{3} / \mathrm{s}$ (compared to the mean minimum annual discharge of $75 \mathrm{~m}^{3} / \mathrm{s}$ ), and there was no flow at all in the Senegal at Bakel for fifteen days.

Further evidence of the severity of this drought was provided by Lake Chad, which shrank to a third of the area and a quarter of the volume it had in 1964 and was divided into two separate lakes by the emergence of the Great Barrier.

This drought is not unique in recent history, since hydrological records for the Senegal at Bamako and the Niger at Koulikoro since the turn of the century show other very severe drought periods in 1910-1914 and 1940-1944, in which the mean annual discharge was lower than in 1969-1973.

Though records before 1930 are very incomplete, the 1913 drought can reasonably be considered to have been similar to the last one. 